Hungarian Delegation

\title{
A new structural version of welded cellular cylindrical shell for a cantilever column
}

\author{
József Farkas $^{1}$, Károly Jármai ${ }^{2}$ \\ ${ }^{1,2}$ University of Miskolc, H-3515 Miskolc, Hungary ${ }^{1}$ Professor emeritus, Dr.sci.techn. \\ altfar@uni-miskolc.hu \\ ${ }^{2}$ Professor, Dr.sci.techn. altjar@uni-miskolc.hu,
}




\section{Abstract:}

A cantilever column is loaded by compression and bending and the horizontal displacement of the column top as well as the outside diameter of the cylindrical shell are limited. The strengthening of the column is performed in the lower part of the column only.

Three structural versions of the column are optimized and compared to each other.

Firstly, the unstiffened circular shell is optimized and it is found that the required large thickness is unsuitable for fabrication.

Secondly, the stringer stiffened circular shell is optimized. The halved rolled UC section stringers are used only in the lower part of the column, the distance of the interruption of stiffeners is also optimized. It is found that the required shell thickness is unsuitable for fabrication.

Thirdly, a new structural version, the cellular shell is used. Cellular shells are constructed from two circular cylindrical shells and a grid of stiffeners welded between them. They have similar advantages than the cellular plates, namely they can produce a large stiffness with small structural height. Their smooth surface is suitable for corrosion protection and they are more aesthetic than the stringer stiffened shells.

The parts of the outer circular shell are welded to the stringers from outer side with longitudinal fillet welds. Halved circular hollow section (CHS) stringers enable the easy welding of the outer fillet welds.

The unknown variables to be optimized are as follows: thicknesses of the inner and outer shell, dimensions and number of the halved CHS stiffeners as well as the distance of the interruption of stiffeners.

The study shows a realistic case when the cellular shell can be used with smaller shell thicknesses and lower cost than the shell stiffened with outer side stringers.

The displacement constraint is so strict that the stress, shell buckling and beam-column buckling constraints are passive.

The cost function to be minimized contents the cost of material, welding and painting. The optimization is performed by a systematic search using a MathCAD algorithm.

Keywords: welded shell structures, structural optimization, cost calculation, cellular structures, cantilever columns, stringer stiffened circular shells, beam-column buckling

\section{Introduction}

Our previous studies have shown that welded cellular plates have some advantages over plates stiffened on one side. Cellular plates can produce large bending and torsional stiffness with a relatively small structural height. Their smooth surface enables a good corrosion protection and advantageous aesthetics.

Welded steel cellular plates can be used for double bottom ships [1,2,3,4] (Williams 1969, Pettersen (1979), Evans and Shanmugam (1981, 1984).

We have published studies on square cellular plates [5,6,7] with halved rolled I section or welded T stiffeners [8,9,10] (Farkas and Jármai 2008,2013) (Farkas 2013) with simply supported edges or supported at four corners [11] (Farkas and Jármai 2013) We have used cellular plates for box column and box beams [12,13] (Jármai and Farkas.2013,2014).

Similar to cellular plates the cellular shells are constructed from two circular cylindrical shells and a grid of stiffeners welded between them (Fig.4). It is advantageous to use halved circular hollow section (CHS) stiffeners, since the parts of the outer circular shell can easily be welded to them. 
The aim of present study is to show the advantages of cellular shells over the stringer stiffened ones. Their large stiffness and small structural height can be useful for a compressed and bent cantilever column in the case of a strict constraint on horizontal displacement of the top together with a constraint on maximum diameter.

The study compares three structural versions for the welded circular cylindrical shell as follows: (a) unstiffened, (b) stiffened with halved rolled I section stiffeners, and (c) stiffened by cellular shell.

The basis of the comparison is the cost, which contents the cost of material, welding and painting.

The base of the column is built-up, but the structural solution of the foundation and its cost is not treated.

Given data: column height $L=15 \mathrm{~m}$, factored compression force $N_{F}=2 \times 10^{7}[\mathrm{~N}]$, horizontal force $H_{F}=0.1 N_{F}$, yield stress of steel $f_{y}=355 \mathrm{MPa}$, elastic modulus $E=2.1 \times 10^{5}$ $\mathrm{MPa}$. In the calculation of displacement the horizontal force is divided by the safety factor $\gamma_{\mathrm{M}}$ $=1.5$.

Constraints: limitation of the horizontal displacement of the column top: $w_{\max }=L / \phi, \phi=1000$ and limitation of the shell diameter: $D=2 R=3000 \mathrm{~mm}$.

\section{The unstiffened shell (Fig.1 without stiffeners)}

The horizontal displacement constraint is formulated as

$$
w_{\max }=\frac{H_{F}}{E I_{1} \gamma_{M}}\left[\frac{\left(L-L_{2}\right)^{3}}{3}+\mu\left(L-\frac{L_{2}}{2}\right)\left(L-L_{2}\right) L_{2}+\mu \frac{L_{2}^{2}}{2}\left(L-\frac{L_{2}}{2}\right)\right] \leq \frac{L}{1000}
$$

where

$$
\begin{gathered}
\mu=\frac{I_{1}}{I_{2}}, \gamma_{M}=1.5 \\
I_{i}=\pi R^{3} t_{i}, i=1,2
\end{gathered}
$$

$I_{\mathrm{i}}$ and $I_{2}$ are the moments of inertia of the upper and lower column part, respectively, $\gamma_{M}$ is the safety factor.

Table 1 shows some results of the systematic search.

Table 1. Some results of the optimization. Dimensions in $\mathrm{mm}$, volume in $\mathrm{mm}^{3}$. he minimum volume is marked by bolt letters

\begin{tabular}{|l|l|l|l|l|}
\hline$t_{1}$ & $t_{2}$ & $L_{2}$ & $w$ & $V \times 10^{-9}$ \\
\hline 36 & 45 & 12000 & 15.00 & 6.107 \\
\hline 35 & 46 & 9000 & 14.94 & 5.881 \\
\hline 24 & 48 & 9000 & 14.93 & 5.429 \\
\hline 18 & 50 & 9000 & 14.88 & $\mathbf{5 . 3 1 6}$ \\
\hline
\end{tabular}


It can be seen that all the solutions need very thick shell parts, not suitable for fabrication. It should be noted that the constraint on beam-column buckling is passive in this case. This constraint is treated in details in section 3.4.

2 The shell stiffened with halved rolled I section stiffeners from outside (Fig.1)

This problem has been treated in [14].

\subsection{Geometric characteristics}

The web height

$h_{1}=h-2 t_{f}$

the cross sectional area

$$
A_{s}=h_{1} \frac{t_{w}}{2}+b t_{f}
$$
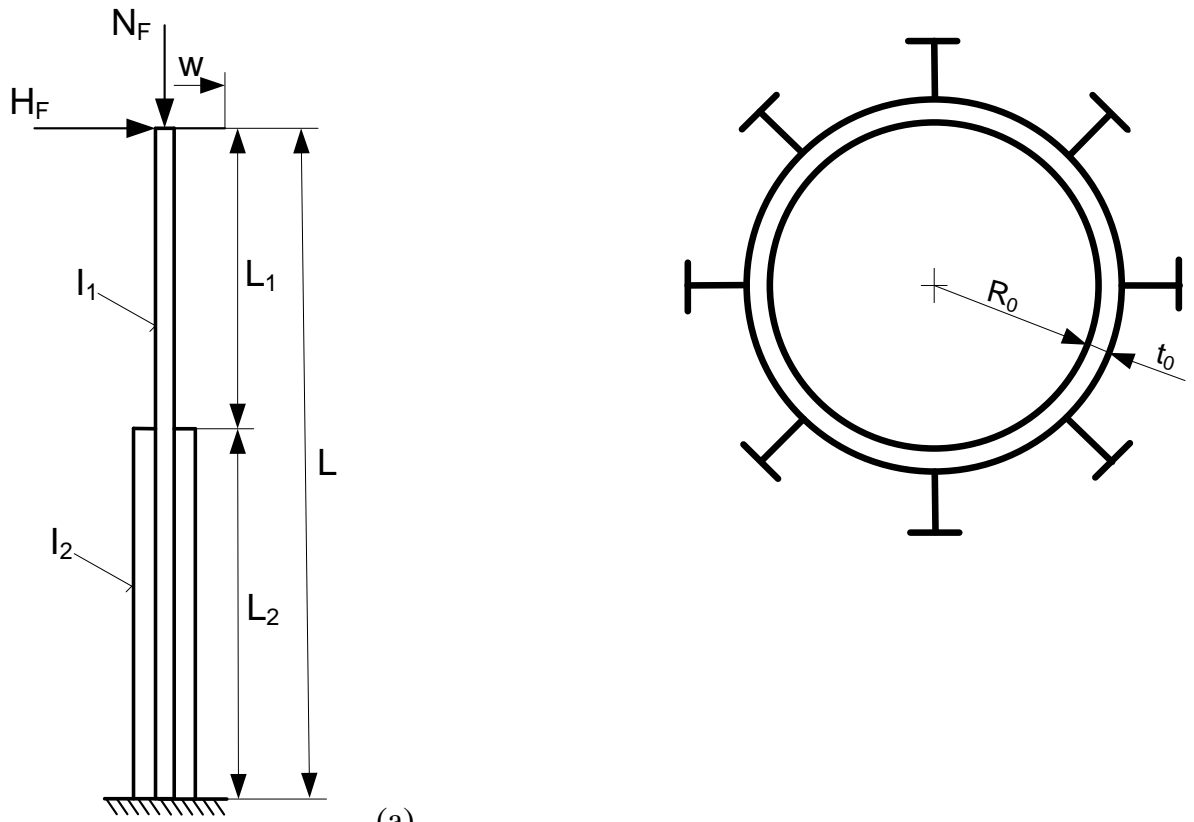

(b)

(a)

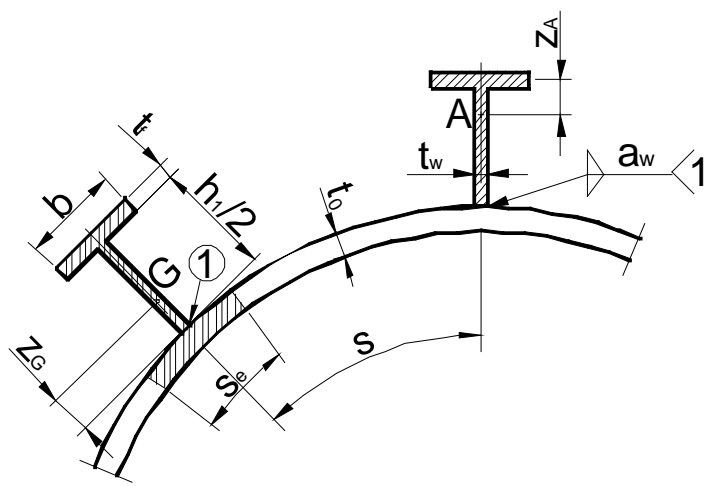

(c) 
Fig.1. (a) Cantilever column loaded by a compressive and a horizontal force, the horizontal displacement $w$ and the shell diameter are limited, (b) cross-section of the column, (c) dimensions of the stringer stiffeners

The distance of the centre of gravity for the halved UC section is

$$
z_{A}=\frac{h_{1} t_{w} / 2\left(h_{1} / 4+t_{f} / 2\right)}{h_{1} t_{w} / 2+b t_{f}}
$$

The moment of inertia of the halved UC section is expressed by

$$
I_{x}=b t_{f} z_{A}^{2}+\frac{t_{w}}{12}\left(\frac{h_{1}}{2}\right)^{3}+\frac{h_{1} t_{w}}{2}\left(\frac{h_{1}}{4}-z_{A}\right)^{2}
$$

The radius of the shell is

$$
R_{0}=R-\frac{h_{1}-t_{f}-t}{2}
$$

\subsubsection{Moment of inertia about an axis of inclined angle}

We need to calculate the moment of inertia about the axis $x$, which encloses an angle $\alpha$ with the axis $\xi$ as well as the moment of inertia about the axis $x^{\prime}$ in a distance $y_{0}$ from axis $x$ (Fig. 2).

The radius in both coordinate system

$$
r^{2}=x^{2}+y^{2}=\xi^{2}+\eta^{2}
$$

Using the triangles GPC and PAC one obtains

$$
\cos \alpha=\frac{x}{\xi+u}, \tan \alpha=\frac{u}{\eta}
$$

From Eqs. $(8,9)$

$$
x=(\xi+\eta \tan \alpha) \cos \alpha=\xi \cos \alpha+\eta \sin \alpha
$$

Using Eq.(10)

$$
x^{2}=\xi^{2} \cos ^{2} \alpha+\eta^{2} \sin ^{2} \alpha+2 \xi \eta \sin \alpha \cos \alpha
$$

Furthermore we use the well-known equation

$$
\sin ^{2} \alpha+\cos ^{2} \alpha=1
$$

With Eqs. $(8,11,12)$ one obtains 
$y^{2}=\xi^{2}+\eta^{2}-\xi^{2} \cos ^{2} \alpha-\eta^{2} \sin ^{2} \alpha-2 \xi \eta \sin \alpha \cos \alpha=\xi^{2} \sin ^{2} \alpha+\eta^{2} \cos ^{2} \alpha-\xi \eta \sin 2 \alpha$

The moment of inertia about the axis $x$ can be calculated as

$I_{x}=I_{\xi} \cos ^{2} \alpha+I_{\eta} \sin ^{2} \alpha-\sin 2 \alpha \int \xi \eta d A$,

since

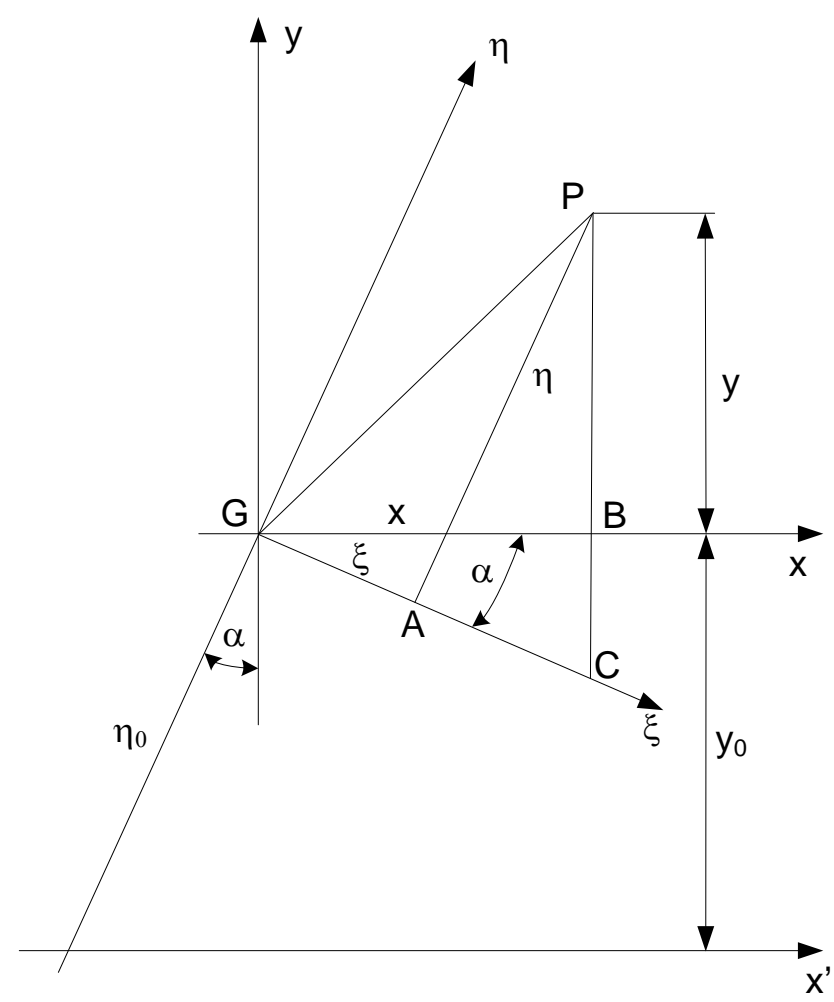

Figure 2. To the moment of inertia about axes of inclined angle ( $x$ and $x$ ')

$I_{\xi}=\int \eta^{2} d A, I_{\eta}=\int \xi^{2} d A$

and

$I_{y}=I_{\xi} \cos ^{2} \alpha+I_{\eta} \sin ^{2} \alpha+\sin 2 \alpha \int \xi \eta d A$

For symmetric cross-sections the third term is zero, thus

$I_{x}=I_{\xi} \cos ^{2} \alpha+I_{\eta} \sin ^{2} \alpha$

The moment of inertia about the axis x' can be calculated as

$I_{x^{\prime}}=\int\left(y+y_{0}\right)^{2} d A=\int y^{2} d A+2 y_{0} \int y d A+y_{0}^{2} \int d A=I_{x}+y_{0}^{2} A$ 
since $\int y d A=0$

When the distance $\eta_{0}$ is known, Eq.(17) can be used as

$$
I_{x^{\prime}}=I_{x}+y_{0}^{2} A=I_{x}+\eta_{0}^{2} \sin ^{2} \alpha A
$$

Using the above derived formulae, the moment of inertia of the whole stiffened shell crosssection is

$$
\begin{aligned}
& I_{x 0}=\pi R_{0}^{3} t+I_{x} \sum_{i=1}^{n_{s}} \cos ^{2}\left(\frac{2 \pi i}{n_{s}}\right)+\left(\frac{h_{1} t_{w}}{2}+b t_{f}\right)\left(R_{0}+\frac{h_{1}+t_{f}}{2}-z_{A}\right)^{2} \sum_{i=1}^{n_{s}} \cos ^{2}\left(\frac{2 \pi i}{n_{s}}\right)+I_{x 01} \\
& I_{x 01}=\left(\frac{b^{3} t_{f}}{12}\right) \sum_{i=1}^{n_{s}} \sin ^{2}\left(\frac{2 \pi i}{n_{s}}\right), q=R_{0}+\frac{h_{1}+t_{f}}{2}-z_{A}
\end{aligned}
$$

It should be mentioned that, in the case of a very strict displacement constraint the effective shell width is $s_{e}=s$.

\subsection{Constraint on horizontal displacement of the column top}

The constraint is the same as for unstiffened shell, see Section 1, Eqs (1), but Eq.(2) is changed to

$$
I_{1}=\pi R_{0}^{3} t_{1} \cdot I_{2}=I_{x 0}
$$

\subsection{Fabrication constraint}

To enable the welding of stiffeners to the shell

$$
n_{\text {s.max }}=\frac{2 R_{0} \pi}{b+300}
$$

\subsection{Cost function}

The cost of welding is formulated according to the fabrication sequence [15].

The general formula for the welding cost is as follows

$$
K_{w}=k_{w}\left(C_{1} \Theta \sqrt{\kappa \rho V}+1.3 \sum_{i} C_{w i} a_{w i}^{n} C_{p i} L_{w i}\right)
$$

where $k_{w}$ [\$/min] is the welding cost factor, $C_{1}$ is the factor for the assembly usually taken as $C_{1}=1$ $\min / \mathrm{kg}^{0.5}, \Theta$ is the factor expressing the complexity of assembly, the first member calculates the time of the assembly, $\kappa$ is the number of structural parts to be assembled, $\rho V$ is the mass of the assembled structure, the second member estimates the time of welding, $C_{w}$ and $n$ are the constants given for the specified welding technology and weld type, $C_{p}$ is the factor of welding position (for downhand 1 , for vertical 2, for overhead 3) 
$L_{w}$ is the weld length, the multiplier 1.3 takes into account the additional welding times (deslagging, chipping, changing the electrode).

(1) Fabrication of 5 shell elements of length $3 \mathrm{~m}$ without stiffeners. For one shell element 2 axial butt welds are needed (GMAW-C) $\left(K_{F 1}\right)$. The cost of forming of a shell element into the cylindrical shape is also included $\left(K_{F 0}\right)$.

(2) Welding of the whole unstiffened shell from 5 elements with 4 circumferential butt welds $\left(K_{F 2}\right)$.

(3) Welding of $n_{s}$ stiffeners to the shell with double-sided GMAW-C fillet welds along $L_{2}$. Number of fillet welds is $2 n_{s}$. $\left(K_{F 3}\right)$.

The material cost is

$$
\begin{aligned}
& K_{M}=k_{M 1} 5 \rho V_{1}+k_{M 2} \rho n_{s} A_{s} L / 2 \\
& V_{1}=3000 \times 2 R \pi t ; \rho=7.85 \times 10^{-6} \mathrm{~kg} / \mathrm{mm}^{3} . k_{F}=1.0 \$ / \mathrm{min}, k_{M 1}=1.0 \$ / \mathrm{kg} .
\end{aligned}
$$

The cost of forming of a shell element of width $3 \mathrm{~m}$ into the cylindrical shape according to (Farkas et al. 2004) is

$$
\begin{aligned}
& K_{F 0}=k_{F} \Theta e^{\mu} ; \mu=6.8582513-4.527217 t^{-0.5}+0.009541996(2 R)^{0.5} \\
& K_{F 1}=k_{F}\left[\Theta \sqrt{\kappa \rho V_{1}}+1.3 \times 0.1520 \times 10^{-3} t^{1.9358}(2 \times 3000)\right]
\end{aligned}
$$

where $\Theta$ is a difficulty factor expressing the complexity of the assembly and $\kappa$ is the number of elements to be assembled

$$
\begin{aligned}
& \kappa=4, V_{1}=2 R_{0} \pi t \times 3000, \Theta=2 \\
& K_{F 2}=k_{F}\left(\Theta \sqrt{25 \rho V_{1}}+1.3 \times 0.1520 \times 10^{-3} t^{1.9358} \times 4 \times 2 R \pi\right) \\
& K_{F 3}=k_{F}\left(\Theta \sqrt{\left(n_{s}+1\right) \rho V_{2}}+1.3 \times 0.3394 \times 10^{-3} a_{w}^{2} 2 L_{2} n_{s}\right)
\end{aligned}
$$

The fillet weld size $a_{w}=0.3 t_{w}, a_{w \min }=3 \mathrm{~mm}$.

$$
V_{2}=5 V_{1}+n_{s} A_{s} L / 2
$$

The cost of painting is

$$
K_{P}=k_{P}\left(4 R \pi L+n_{s} L_{2} S\right), S=h_{1}+2 b, k_{P}=14.4 \times 10^{-6} \$ / \mathrm{mm}^{2}
$$

The total cost is

$$
K=K_{M}+5 K_{F 1}+5 K_{F 0}+K_{F 2}+K_{F 3}+K_{P}
$$

\subsection{Results of the optimization}


Some results of the systematic search are given in Table 2 .

Table 2. Some results of the optimization. Dimensions in $\mathrm{mm}$, volume in $\mathrm{mm}^{3}$ and costs in $\$$. The optima are marked by bolt letters

\begin{tabular}{|c|c|c|c|c|}
\hline$h$ & 222.2 & 222.2 & 161.8 & 152.4 \\
\hline$b$ & 209.1 & 208.1 & 154.4 & 152.2 \\
\hline$t_{w}$ & 12.7 & 12.7 & 8 & 5.8 \\
\hline$t_{f}$ & 20.5 & 20.5 & 11.5 & 6.8 \\
\hline$t$ & 45 & 46 & 42 & 42 \\
\hline$n_{s}$ & 18 & 18 & 20 & 20 \\
\hline$L_{2}$ & 12000 & 9000 & 9500 & 9000 \\
\hline$w$ & 14.99 & 14.99 & 14.84 & 14.96 \\
\hline $10^{-9} V$ & 7.0130 & 6.847 & 6.666 & $\mathbf{6 . 5 4 5}$ \\
\hline$K$ & 96480 & 94850 & 88500 & $\mathbf{8 8 0 1 0}$ \\
\hline
\end{tabular}

The constraints on panel buckling and beam-column buckling (see section 3.3) are passive.

It can be seen that the decrease of $L_{2}$ and the dimensions of stiffeners gives less volume and cost.

The main problem is the large shell thickness (over $40 \mathrm{~mm}$ ), which is unsuitable for fabrication.

\section{The column stiffened by cellular shell (Figs.3,4)}

Halved circular hollow section (CHS) [16] stiffeners are used. This type of stiffeners have more advantages as follows: (a) they enable suitable welded joints for the cover plate elements, (b) their large torsional stiffness gives a large overall stiffness for the whole structure.

\subsection{Geometric characteristics}

The cross-sectional area of a half CHS is (Fig.3)

$$
A_{s}=\pi R_{s} t_{s}, R_{s}=\frac{D_{s}-t_{s}}{2}
$$

the distance of its gravity centre is

$$
y_{G}=\frac{2 R_{s}}{\pi}
$$




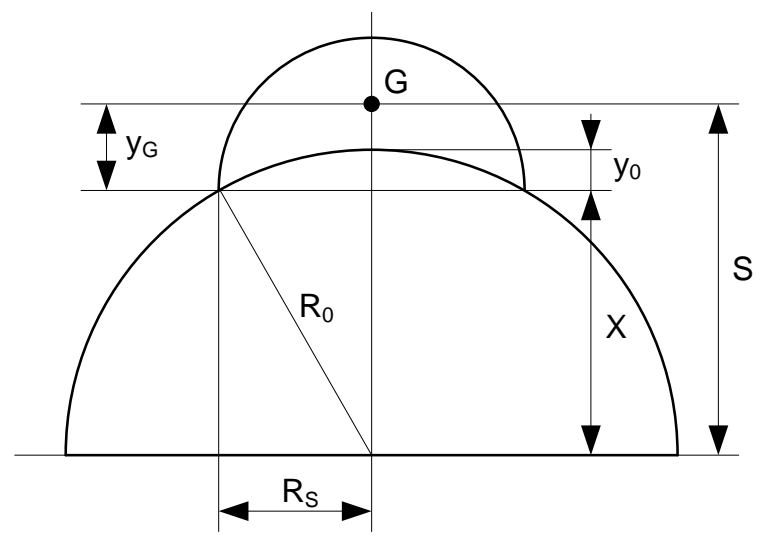

Fig.3. Geometry of the cellular shell

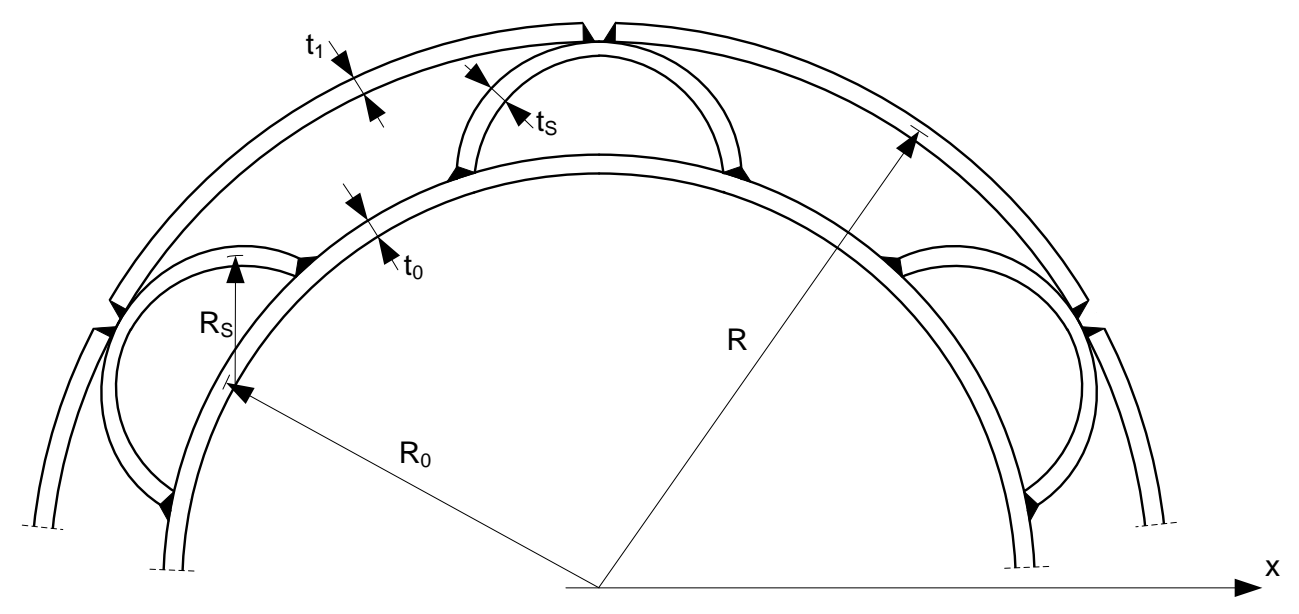

Fig.4. Dimensions of a cellular shell

and its moment of inertia

$$
I_{s}=\frac{R_{s}^{3} \pi}{2}\left(1-\frac{8}{\pi^{2}}\right)
$$

The distance of its gravity centre to the shell centre

$$
s=y_{G}+X, X=\sqrt{R_{0}^{2}-R_{s}^{2}}, y_{0}=R_{0}-X
$$

The radius of the inner shell $R_{0}$ can be calculated from the following equation

$$
R=s+\frac{t_{s}+t_{1}}{2}
$$

( $t_{1}$ is the thickness of the outer shell) 


$$
R_{0}=\sqrt{\left(R-\frac{2 R_{s}}{\pi}-R_{s}-\frac{t_{s}+t_{1}}{2}\right)^{2}+R_{s}^{2}}-\frac{t_{0}}{2}
$$

The moment of inertia of $n_{s}$ stiffeners about the centre of the shell using Eqs (16) and (18)

$$
I_{x s}=\left(I_{s}+A_{s} s^{2}\right) \sum_{i=1}^{n_{s}} \cos ^{2}\left(\frac{2 \pi i}{n_{s}}\right)+\left(\frac{\pi R_{s}^{3} t_{s}}{2}\right) \sum_{i=1}^{n_{s}} \sin ^{2}\left(\frac{2 \pi i}{n_{s}}\right)
$$

The moment of inertia of the whole cellular shell (Fig.4)

$$
I_{x}=\pi R_{0}^{3} t_{0}+\pi R^{3} t_{1}+I_{x s}
$$

Fabrication constraint to enable the welding of the half CHS to the inner shell:

from

$$
\frac{2 R_{0} \pi}{n_{s}}-2 R_{s} \geq 2 t_{s}
$$

the maximum allowable number of half CHS stiffeners

$$
n_{\text {s.max }}=\frac{\pi R_{0}}{R_{s}+t_{s}}
$$

\subsection{Constraint on horizontal displacement of the column top}

This constraint is the same as in Section 1, see Eq.(1), but Eq.(2) is changed to

$$
I_{1}=\pi R_{0}^{3} t_{1}, I_{2}=I_{x}
$$

\subsection{Constraint on panel shell buckling of the outer shell parts between stiffeners}

According to the Det Norske Veritas [17] design rules for shell buckling

$$
\begin{aligned}
& \sigma_{\max }=\frac{N_{F}}{A}+\frac{H_{F} L}{W_{x}} \leq f_{k s}=\frac{f_{y}}{\sqrt{1+\bar{\lambda}^{4}}}, \bar{\lambda}=\sqrt{\frac{f_{y}}{f_{E}}} \\
& f_{E}=C \frac{\pi^{2} E}{12\left(1-v^{2}\right)}\left(\frac{t_{1}}{s_{0}}\right)^{2}, C=\psi \sqrt{1+\left(\frac{\rho_{0} \xi}{\psi}\right)^{2}} \\
& \psi=4, \rho_{0}=0.5\left(1+\frac{R}{150 t_{1}}\right)^{-0.5}, \xi=0.702 Z
\end{aligned}
$$




$$
Z=\frac{s_{0}^{2}}{R t_{1}} \sqrt{1-v^{2}}, v=0.3
$$

In the case of such a very strict displacement constraint the panel buckling constraint is not active. $\sigma_{\max }$ is so small that the effective shell width is equal to the whole width $s_{0}$.

Calculations show that this constraint is not active.

\subsection{Constraint on beam-column buckling}

The check should be performed by taking into account the overall buckling of the column. For the calculation of the Euler critical stress the formula given by Timoshenko and Gere [18] is used.

$$
\begin{aligned}
& \frac{N_{F}}{\chi_{1} A f_{y}}+k_{y y 1} \frac{H_{F} L}{W_{x} f_{y}} \leq 1 \\
& \chi_{1}=\frac{\phi_{1}-\sqrt{\phi_{1}^{2}-\lambda^{2}}}{2 \lambda^{2}}, \phi_{1}=1+\alpha(\lambda-0.2)+\lambda^{2}, \lambda=\sqrt{\frac{f_{y}}{\sigma_{E}}}, \sigma_{E}=\frac{\pi^{2} E I_{2}}{4 L^{2} A C} \\
& C=\frac{L_{2}}{L}+\frac{L_{1} I_{2}}{L I_{1}}-\frac{1}{\pi}\left(\frac{I_{2}}{I_{1}}-1\right) \sin \frac{\pi L_{2}}{L} \\
& \alpha=0.34, \\
& k_{y y 1}=C \cdot_{m y}\left(1+0.6 \lambda \frac{F}{\chi_{1} A f_{y}}\right) \quad \text { for } \lambda<1 \\
& k_{y y 1}=C \cdot\left(1+0.6 \frac{F}{\chi_{1} A f_{y}}\right) \text { for } \lambda \geq 1 \\
& C_{m y}=0.6
\end{aligned}
$$

\subsection{The cost function}

The first part, i.e. the fabrication of the base shell, is the same as for the stiffened shell (Section 2.4), but $R$ and $t$ should be changed to $R_{0}$ and $t_{0}$

$$
\begin{aligned}
& V_{1}=3000 \times 2 R_{0} \pi t_{0}, \rho=7.86 \times 10^{-6} \mathrm{kgmm}^{-3}, k_{F}=1.0 \$ / \mathrm{min}, k_{M 1}=1.0 \$ / \mathrm{kg} . \\
& K_{F 0}=k_{F} \Theta e^{\mu}, \mu=6.8582513-4.527217 t_{0}^{-0.5}+0.009541996\left(2 R_{0}\right)^{0.5} \\
& \left.K_{F 1}=k_{F} \mid \Theta \sqrt{\kappa \rho V_{1}}+1.3 \times 0.152 \times 10^{-3} t_{0}^{1.9358}\left(6000+2 \pi R_{0}\right)\right]
\end{aligned}
$$




$$
\begin{aligned}
& \kappa=4, V_{1}=2 R_{0} \pi t_{0} \times 3000, \Theta=2 \\
& K_{F 2}=k_{F}\left(\Theta \sqrt{25 \rho V_{1}}+1.3 \times 0.152 \times 10^{-3} t_{0}^{1.9358} \times 4 \times 2 R_{0} \pi\right)
\end{aligned}
$$

Welding of the half CHS stiffeners to the base shell using SAW fillet welds

$$
\begin{aligned}
& K_{F 3}=k_{F}\left\lfloor\Theta \sqrt{\left(n_{s}+1\right) \rho V_{2}}+1.3 \times 0.2349 \times 10^{-3} a_{w}^{2} 2 L_{2} n_{s}\right\rfloor \\
& V_{2}=5 V_{1}+n_{s} A_{s} L_{2}, \quad a_{w}=0.3 t_{s}\left(a_{\min }=3 \mathrm{~mm}\right)
\end{aligned}
$$

Forming of the outer curved shell panels of length $3 \mathrm{~m}$

$$
K_{F 4}=k_{F} \Theta e^{\mu_{1}}, \mu_{1}=6.8582513-4.527217 t_{1}^{-0.5}+0.009541996(2 R)^{0.5}
$$

Welding of an outer curved shell panel of length $L_{2}$ using $\kappa_{3}$ shell parts of length $3 \mathrm{~m}$ by $\left(\kappa_{30}-1\right)$ GMAW-C butt welds

$$
\begin{aligned}
& K_{F 5}=k_{F}\left(\Theta \sqrt{\kappa_{30} \rho V_{3}}+1.3 \times 0.152 \times 10^{-3} t_{1}^{1.9358} \frac{2 R \pi}{n_{s}}\left(\kappa_{30}-1\right)\right) \\
& V_{3}=\frac{2 R \pi t_{1} L_{2}}{n_{s}} \\
& \kappa_{3}=\frac{L_{2}}{3000} \text { rounded up to } \kappa_{30}
\end{aligned}
$$

Welding of the outer panels to the stiffened shell by SAW fillet welds of size $a_{w 1}=0.3 t_{s}$

$$
\begin{aligned}
& K_{F 6}=k_{F}\left(\Theta \sqrt{\left(n_{s}+1\right) \rho V_{4}}+1.3 \times 0.2349 \times 10^{-3} a_{w 1}^{2} 2 n_{s} L_{2}\right) \\
& V_{4}=V_{2}+n_{s} V_{3}
\end{aligned}
$$

Painting cost

$$
K_{P}=k_{p} S, S=2 \pi R_{0}\left(2 L-L_{2}\right)+2 R \pi L_{2}
$$

Material cost

$$
K_{M}=k_{M} \rho V_{4}
$$

The total cost

$$
K=K_{M}+5\left(K_{F 0}+K_{F 1}\right)+K_{F 2}+K_{F 3}+\kappa_{30} K_{F 4}+n_{s} K_{F 5}+K_{F 6}+K_{P}
$$




\subsection{Results of the optimization}

The optima are found by a systematic search using a MathCAD algorithm.

The details of the search are shown in Table 3. For fabrication aspects the following limits are introduced: $t_{0 \max }=30, t_{1 \max }=30, t_{\sin }=10 \mathrm{~mm}$.

Table 2. Details of the optimization. The maximal displacement in each case is near the allowable value of $15 \mathrm{~mm}$. Dimensions in $\mathrm{mm}$, volume $V$ in $\mathrm{mm}^{3}$. The optimum is marked by bold letters

\begin{tabular}{|c|c|c|c|c|}
\hline$t_{0}$ & $t_{1}$ & $L_{2}$ & $V_{4} \times 10^{-9}$ & $K(\$)$ \\
\hline 25 & 29 & 8900 & 5.768 & 78460 \\
\hline 24 & 30 & 8900 & 5.720 & $\mathbf{7 7 4 4 0}$ \\
\hline 23 & 30 & 9300 & 5.705 & 79410 \\
\hline 22 & 30 & 9800 & 5.719 & 78740 \\
\hline 21 & 30 & 10300 & 5.733 & 78100 \\
\hline 20 & 30 & 10900 & 5.776 & 77740 \\
\hline 19 & 30 & 11700 & 5.876 & 77940 \\
\hline 18 & 30 & 13200 & 6.1780 & 82920 \\
\hline
\end{tabular}

The numerical values show the following results:

(a) The minimum volume and cost is found for the minimum number of stiffeners $n_{s}=4$ :

(b) the CHS stiffener profile of $101.6 \times 10$ gives the minimum volume and cost. The tendency is to minimize $D_{s}$ to maximize $R_{0}$, and to maximize $t_{s}$, thus, we select this profile.

\section{Conclusions}

A realistic numerical problem is investigated, in which the outer shell diameter and the horizontal displacement of the column top are limited. The numerical value of the compression force $N_{F}$ is also given. Three structural solutions are optimized:

(1) unstiffened circular cylindrical shell has the minimal volume $V=5.316 \times 10^{9} \mathrm{~mm}^{3}$, but the shell thickness is $50 \mathrm{~mm}$, which is unsuitable for fabrication,

(2) circular shell stiffened with halved rolled UC sections has the structural volume $V=$ $6.545 \times 10^{9} \mathrm{~mm}^{3}$ and the cost $K=88010 \$$, but the shell thickness is $42 \mathrm{~mm}$, unsuitable for fabrication,

(3) optimum solution of the column strengthened by cellular shell has values $V=$ $5.705 \times 10^{9} \mathrm{~mm}^{3}$ and $K=77440 \$$, thickness $30 \mathrm{~mm}$.

It can be concluded that in this case only the cellular shell can fulfil all the requirements (horizontal displacement, maximum outer diameter, maximum thickness of $30 \mathrm{~mm}$, minimum cost). In addition, the cellular shell can be more easily protected against corrosion and is much more aesthetic than the second version.

\section{Acknowledgements}

The research was supported by the Hungarian Scientific Research Fund OTKA T 109860 projects and was partially carried out in the framework of the Center of Excellence of Innovative Engineering Design and Technologies at the University of Miskolc. 


\section{References}

[1] Williams,D.G. 1969. Analysis of doubly plated grillage under inplane and normal loading. Ph.D.thesis. Imperial College, London.

[2] Pettersen,E. 1979. Analysis and design of cellular structures. University of Trondheim, Norwegian Institute of Technology.

[3] Evans,H.R., Shanmugam,N.E., 1984. Simplified analysis for cellular structures. J. Struct. Eng. ASCE, 1984, 110, 531-543.

[4] Shanmugam,N.E.,. Evans,H.R., A grillage analysis of the nonlinear and ultimate load behavior of cellular structures under bending loads. Proc. Inst. Civ. Eng. Part 2. 1984, 71, 705-719

[5] Farkas,J., Structural synthesis of welded cell-type plates. Acta Techn. Hung.1976, 83, No. 1-2, 117-131.

[6] Farkas,J., Optimum design of metal structures, Akadémiai Kiadó, Budapest, Ellis Horwood, Chichester, UK, 1984

[7] Farkas,J., Jármai,K., Analysis and optimum design of metal structures. Balkema, Rotterdam-Brookfield, 1997.

[8] Farkas,J., Jármai,K., Design and optimization of metal structures,. Horwood, Chichester, UK, 2008

[9] Farkas,J., Jármai,K., Optimum design of steel structures, Springer, Heidelberg etc., 2013

[10] Farkas,J., Minimum volume and cost design of a welded square cellular plate with welded T-stiffeners. In „Design, Fabrication and Economy of Metal Structures, Proc. Int.Conf. Miskolc, 2013. Eds Jármai,K., Farkas,J. Springer, Heidelberg etc. 2013” 3-9.

[11] Farkas,J., Jármai,K.: Cost comparison of optimized welded square cellular plates supported at four corners with two different kinds of stiffeners. IIW-doc. XV-1441-13, XVF-91-13. 10p.

[12] Jármai,K., Farkas,J., Comparison of rectangular and square box columns constructed from cellular plates with welded and rolled stiffeners. Eng Struct, 2014, 60, 199-205

[13] Jármai,K., Farkas,J., Minimum cost design of a belt-conveyor bridge constructed as a box beam with welded cellular walls, IIW Int.Conf.2014. Seoul.

[14] Farkas,J., Jármai,K. Optimum design of a welded stringer-stiffened steel cylindrical shell subject to axial compression and bending. IIW-Doc. XV-1167-04, XV-WG9-26 -04, Osaka, 2004

[15] Jármai,K., Farkas,J. Cost calculation and optimization of welded steel structures. J Constr Steel Res, 1999, 50, 115-135

[16] EN 10219-2. Cold formed circular hollow section profiles. CEN 2006

[17] DNV Det Norske Veritas, Buckling strength of shells. Recommended practice RP-C202. Høvik, Norway, 2002

[18] Timoshenko,S.P., Gere,J.M. Theory of elastic stability. McGraw Hill. New YorkToronto-London, 1961. 